\section{Detection of Sabin-Like Type 2 Poliovirus from Sewage After Global Cessation of Trivalent Oral Poliovirus Vaccine - Hyderabad and Ahmedabad, India, August-September 2016}

Sunil Bahl, MD ${ }^{1}$; Lee M. Hampton, $\mathrm{MD}^{2}$; Pankaj Bhatnagar, $\mathrm{MD}^{3}$; Gadala Srinivasa Rao, MD ${ }^{4}$; Pradeep Haldar, MBBS ${ }^{5}$; Lucky Sangal, $\mathrm{MD}^{3}$; Puttaraju AK Jetty, $\mathrm{MBBS}^{3}$; Uma P Nalavade, $\mathrm{MSc}^{6}$

During September 2-October 4, 2016, four sewage samples collected during August 3-September 19 (Hyderabad, Telangana State, India) and one sewage sample collected on August 30 (Ahmedabad, Gujarat State, India) tested positive for Sabin-like type 2 polioviruses. These polioviruses were detected approximately 4 months after April 25, 2016, when India officially ceased use of trivalent oral poliovirus vaccine (tOPV), containing Sabin attenuated types 1, 2, and 3 polioviruses, and switched to bivalent OPV (bOPV), containing Sabin attenuated types 1 and 3 polioviruses (1).

Detection of Sabin-like type 2 poliovirus approximately 4 months after the switch from tOPV to bOPV suggested that tOPV use might have continued after it was supposed to stop globally, creating a risk for emergence of new type 2 vaccinederived polioviruses (VDPV2s), which can cause paralysis. Genetic sequencing of the 903-nucleotide VP1 region of the isolated viruses showed zero, one, two, and four nucleotide changes in the four Hyderabad isolates and one nucleotide change in the Ahmedabad isolate, compared with the type 2 polioviruses in tOPV. These findings indicated that the isolated polioviruses had not replicated sufficiently to accumulate more than a few mutations on a potential pathway to becoming VDPV2s, and that the tOPV they originated from had likely been used during the preceding 4 months.

In accordance with global guidelines for responding to poliovirus events (2), detailed investigations were initiated within 48 hours of detection of the type 2 poliovirus in Hyderabad and the neighboring Rangareddy district, and in Ahmedabad (Box). As part of global poliovirus containment efforts (3), laboratories in those areas potentially storing type 2 polioviruses had previously been found to not have such polioviruses, so they were not searched. Telangana and Gujarat state officials met with immunization program stakeholders in the affected districts and other districts in their states regarding the need to reconfirm withdrawal of all tOPV.

In Hyderabad and Rangareddy districts, the two main district vaccine cold stores, along with 13 private vaccine retailers and distributors and 4,498 public and private health facilities, were searched during September 4-October 5. Thirty-seven tOPV vials from four manufacturers were found in 17 private clinics; the majority were small clinics not affiliated with an organized medical association. Twenty-two of the tOPV vials were unopened; however, 15 had been partially used. Six vials were beyond their expiration date, and 31 had expiration dates from December 2016 to November 2017. No tOPV vials or bulk type 2 polio vaccine were found at the only vaccine manufacturer in Hyderabad.

In Ahmedabad District, the main district vaccine cold store, 572 other cold chain storage points and public and private health facilities, and 12 private vaccine retailers and distributors were searched during September 14-October 17. Two tOPV vials were found at a private vaccine retailer, and another 11 tOPV vials were found at eight private clinics; the majority were small clinics not affiliated with an organized medical association. All tOPV vials had expiration dates ranging from December 2016 to November 2017.

BOX. Components of investigations to find trivalent oral poliovirus vaccine (tOPV) still in use after its use was officially ceased Hyderabad and Ahmedabad, India

- Conduct immediate search of all known vaccine cold chain storage points.

- Visit all health facilities regularly reporting acute flaccid paralysis cases to inquire about tOPV use, with extensive search for $\mathrm{tOPV}$ vials at medical colleges and other large health facilities.

- Conduct a street-by-street physical check of all public and private health facilities that do not regularly report acute flaccid paralysis cases.

- Map and search all private vaccine retailers and distributors in coordination with the state drug regulator.

- Upon identification of any tOPV vials at any location, trace back the source and timing of the supply. Ensure safe disposal of recovered vaccine.

- Visit any OPV manufacturers to check for tOPV and bulk type 2 polio vaccine.

- Meet with immunization program stakeholders (e.g., professional health associations, public and private hospitals, vaccine retailers and distributors, immunization program officers, cold chain officers, and World Health Organization and United Nations Children's Fund staff members) regarding the need to reconfirm tOPV withdrawal. 
All tOPV vials found during the investigations had been purchased and delivered before the switch from tOPV to bOPV. All tOPV vials found were removed, labeled for destruction, and placed in the responsible immunization officer's custody.

These investigations for tOPV possibly in use after the global switch from tOPV to bOPV are the first triggered by detection of Sabin-like type 2 polioviruses in either environmental surveillance sewage samples or stool specimens from persons with paralysis. The finding of $\mathrm{tOPV}$ vials in health facilities and at a vaccine retailer indicates that some tOPV might still be in use and that future detections of Sabin-like type 2 virus anywhere should prompt checks for tOPV vials, according to the guidelines for responding to type 2 polioviruses (2). The risk that Sabin-like type 2 virus could spread and evolve into a circulating VDPV2 increases over time with the progressive decrease in population immunity to type 2 poliovirus infection following the switch from tOPV to bOPV (4).

The finding that all tOPV discovered was at a private vaccine retailer or private clinics indicates that future investigations to identify tOPV still in use should carefully assess the private sector. This investigation underscores the importance of maintaining robust surveillance for polioviruses and of immunization workers being alert for tOPV vials in cold chain storage and reporting any tOPV vials that they find. Additional efforts are needed to ensure that the private sector is aware of the need for cessation of tOPV use.

\footnotetext{
${ }^{1}$ World Health Organization, South-East Asia Regional Office, New Delhi, India; ${ }^{2}$ Global Immunization Division, CDC; ${ }^{3}$ World Health Organization, Country Office for India, New Delhi, India; ${ }^{4}$ Government of Telangana, India; ${ }^{5}$ Ministry of Health and Family Welfare, Government of India, New Delhi, India; ${ }^{6}$ Enterovirus Research Centre, Mumbai, India.
}

Corresponding author: Lee M. Hampton, lhampton@cdc.gov, 404-639-4722.

\section{Acknowledgments}

C. Shivleela, Institute of Preventive Medicine, Hyderabad; Disha Patel, BJ Medical College, Ahmedabad; Deepa Sharma, Enterovirus Research Centre, Mumbai; Rajeshwar Tiwari, Budhaprakash Jyoti, G. Sudheera, S. Prasanna Kumari, Ganesh Rao, Government of Telangana; N.P. Jani, Government of Gujarat, Asish Satapathy, B.P. Subramanya, Raman Sethi, Vikas Kokare, Aniket Rana, C.R. Patel, Pragath Kumar, Atul P Nagime, N Veera Bhadrudu, National Polio Surveillance Project, World Health Organization, India.

\section{References}

1. Hampton LM, Farrell M, Ramirez-Gonzalez A, et al.; Immunization Systems Management Group of the Global Polio Eradication Initiative. Cessation of trivalent oral polio vaccine and introduction of inactivated poliovirus vaccine-worldwide, 2016. MMWR Morb Mortal Wkly Rep 2016;65:934-8. http://dx.doi.org/10.15585/mmwr.mm6535a3

2. Global Polio Eradication Initiative. Responding to a poliovirus event and outbreak-part 2: protocol for poliovirus type 2. Geneva, Switzerland: World Health Organization, Global Polio Eradication Initiative; 2016. http://polioeradication.org/wp-content/uploads/2016/09/ Responding-to-a-poliovirus-event-and-outbreak-SOPs-Part-2-Protocolfor-PV-Type-2_EN.pdf

3. Previsani N, Tangermann RH, Tallis G, Jafari HS. World Health Organization guidelines for containment of poliovirus following typespecific polio eradication —-worldwide, 2015. MMWR Morb Mortal Wkly Rep 2015;64:913-7. http://dx.doi.org/10.15585/mmwr.mm6433a5

4. Duintjer Tebbens RJ, Hampton LM, Thompson KM. Implementation of coordinated global serotype 2 oral poliovirus vaccine cessation: risks of inadvertent trivalent oral poliovirus vaccine use. BMC Infect Dis 2016;16:237. http://dx.doi.org/10.1186/s12879-016-1537-8 